\title{
Effect of Degree of Substitution on the Adhesive Propterties of Methyl Cellulose Derived from Waste Bitter Orange [Citrus Aurantium (Linn.)] Mesocarp
}

\author{
1*Hamza Abba \\ ${ }^{2}$ Abdulqadir Ibrahim \\ ${ }^{1}$ Sani Uba \\ ${ }^{3}$ Isuwa Kari
}

\author{
${ }^{1}$ Department of Chemistry, Ahmadu Bello University, P.M.B.1045, Zaria, Nigeria \\ ${ }^{2}$ Department of Chemistry, Nigeria Police Academy, P.M.B.3474, Wudil, Kano, Nigeria \\ ${ }^{3}$ Nigerian Institute of Leather Science and Technology, P.M.B. 1034, Zaria, Nigeria
}

\section{Doi:10.5901/ajis.2013.v2n13p19}

\begin{abstract}
Cellulose was extracted from waste bitter orange mesocarp and chemically modified by etherification in propan-2-ol as solvent with varying amount of methyl chloride and the degree of substitution (DS) of the methyl group for $\mathrm{H}$ of the native cellulose calculated by acid-base titration. Nine pastes (seven etherified cellulose (methyl cellulose) and the native cellulose (control) were prepared by dispersing them in distilled water (35\% w/v) and heating the eight dispersions to $45^{\circ} \mathrm{C}$ with continuous agitation for $15 \mathrm{~min}$. The solutions were then cooled to room temperature and allowed to gel. The effect of DS on the adhesive properties of the pastes (tack strength, rolling ball test, gelation time, drying time, optical clarity, relative viscosity, syneresis) was comparatively investigated. The results showed that the DS in the etherified cellulose ranged from 1.31 to 1.98 with the methyl cellulose giving higher values of viscosity and tack strength with increase in DS. However, lower values of syneresis, gelation time, optical clarity, drying time and rolling ball distance were recorded with increase in DS. Increase in desirable adhesive properties of the methyl cellulose is attributed to its relatively higher molecular weight compared to the native cellulose. Production and use of etherified cellulose, in place of petroleum-based synthetic adhesives, in remoistening applications such as envelope flaps, postage stamps, labels and other paper works in Nigeria and other developing economies is recommended from the viewpoints of economy, environment, health and safety.
\end{abstract}

Keywords: Adhesion, cellulose, degree of substitution, tack strength, viscosity

\section{Introduction}

Adhesion, attachment between an adhesive and a substrate, is the molecular attraction exerted between bodies in contact. An adhesive is any substance that, when applied to the surfaces of materials, binds the surfaces together and resists separation (Mittal and Pizzi, 2003; Notley and Norgren, 2006; Ebnesajjad, 2010). Use of adhesives offers many advantages over other binding techniques such as sewing, welding, bolting and screwing (Forsström et al., 2005; Nolte et al., 2009). These advantages include the ability to bind different materials together and distribute stress more efficiently across the joint, cost effectiveness as an easily mechanized process, improvement in aesthetic design, and increase in design flexibility (Maeda et al., 2002; Chen et al., 
2005; Huang et al., 2005; Eriksson et al., 2007; Pettersson and Dedinaite, 2008). Carbohydrates, in the form of polymers such as cellulose, starch, and natural gums, are available in large quantities from various plant sources. Each of them has potentials for utilization as adhesive and in adhesive formulations (McMurry, 2012). Bioadhesives are preferred to petroleum-derived synthetic adhesives from the points of view of economy, environment, health and safety (Doraiswamy et al., 2009; Blackman et al., 2012). Cellulose is considered to be a natural condensation polymer of 2000 to $14000 \beta$-1,4-glycosidically linked glucose residues, each carrying three reactive hydroxyl groups (Klemm et al., 2005; Carraher, J r, 2010; Karabulut and Wågberg, 2011):

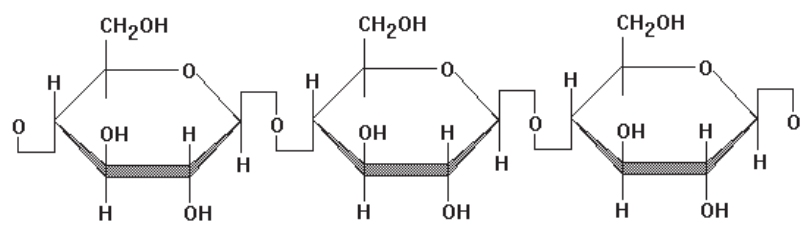

Fig. 1. Glucose Residues in Cellulose (adapted from Klemm et al., 2005)

Replacing one or more of the cellulose's hydroxyls with other functional groups results in production of cellulose derivatives with desired functional properties (Ito et al., 2007). Etherification, the process of making ether from alcohol, is an efficient method of modifying properties of polysaccharides, such as cellulose (Ahola et al., 2008; Fox et al., 2011). $\left[\mathrm{C}_{6} \mathrm{H}_{7} \mathrm{O}_{2}(\mathrm{OH}) \times\left(\mathrm{OCH}_{3}\right)_{y}\right]_{n}$ is the condensed molecular formula of methyl cellulose, whose structural formula is (Carey, 2008; Henriksson et al., 2008; Villetti et al., 2011):

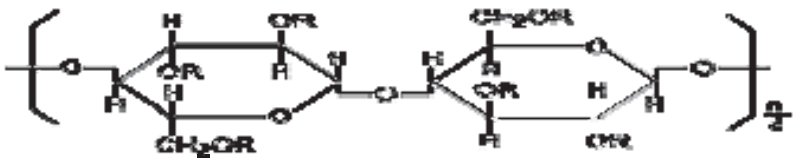

Fig. 2. Methyl Cellulose, $\mathrm{R}=\mathrm{CH}_{3}$ (adapted from Villetti et al., 2011 )

By varying both the length of the polymer backbone and the number of substitutive alkyl groups, derivatives of a wide range of modified cellulose grades can be manufactured to provide different levels of viscosity and tuning the several properties of the polymer for adhesive use. Thus, control of degree of substitution (DS) and uniform distribution of functional groups along the polymer's chain have been the major challenges confronting synthetic polymer chemists (Elomaa, 2004). Although the maximum DS for a glucose molecule would be 3 , this is not practically possible to achieve because of other competing side reactions (Samios et al., 1997). Amim and colleagues (2009) have reported average DS values between 1.3-2.6 for methyl cellulose with methoxy substitution between 27.5-31.5\% (weight). Mario and associates (2005) have also reported 1.5-1.9 as the average number of substituent groups (DS) for sodium carboxymethyl cellulose. Bitter orange (Citrus aurantium) tree is a hybrid between Citrus maxima (pomelo) and Citrus reticulata (mandarin) that is spiny and evergreen (Rodrigues et al., 2013). Many varieties of bitter orange are used for their essential oils in perfumery, as flavouring and as solvent, employed in herbal medicines as stimulant and appetite suppressant, due to its active ingredient, synephrine (Jordan et al., 2004). However, I ts supplements have, recently, been linked to a number of serious side effects and deaths to consumers of its fruit (Hess and Sullivan, 2005). The aim of this study was to extract cellulose from waste bitter orange mesocarp, chemically modify the native cellulose by adding varying amount of methyl chloride of varying degree of substitution and comparatively 
investigate suitability of the products for remoistenable adhesive use in postage stamps, envelope flaps, labels and other paper works.

\section{Methods and Procedures}

Waste bitter orange (Citrus aurantium) mesocarp was obtained from local orange sellers at Samaru Market, Sabon Gari Local Government, Kaduna State. Analytical-grade reagents phenolphthalein, $\mathrm{NaOH}, \mathrm{HCl}$, ppropan-2-ol, ethanol, and methyl chloride were purchased from Sigma Aldrich (Munich, Germany) and used as supplied.

\subsection{Extraction of Cellulose and its Derivatization to MC}

Cellulose was extracted with $8 \% \mathrm{NaOH}$ from the mesocarp of waste bitter orange according to the procedure reported by Pushpamalar et al., 2006. The cellulose obtained was etherified to eight different MC products by adding $0.25,0.30,0.35,0.40,0.45,0.50,0.55$ and $0.60 \mathrm{~g}$ of methyl chloride to $2 \mathrm{~g}$ of the native cellulose powder and heating the product to $27^{\circ} \mathrm{C}$ for $2 \mathrm{~h}$ in $20 \mathrm{~mL}$ of $15 \%$ in $20 \mathrm{~mL}$ propan-2-ol.The product obtained was thoroughly washed with $75 \%$ ethanol and dried at $60^{\circ} \mathrm{C}$ in oven.

\subsection{Determination of Degree of Substitution:}

Each sample $(0.5 \mathrm{~g})$ was placed in a $250-\mathrm{ml}$ conical flask. Distilled water $(50 \mathrm{ml})$ was added and the $\mathrm{pH}$ adjusted to 7.0 with $0.02 \mathrm{M} \mathrm{HCl} .0 .5 \mathrm{M} \mathrm{NaOH}(25 \mathrm{ml})$ was introduced and the mixture heated on hot plate until a transparent solution was obtained. The excess $\mathrm{NaOH}$ was titrated with $\mathrm{HCl}$ (using phenolphthalein as indicator) back to $\mathrm{pH}$ 7.0. Triplicate preparations were performed on each

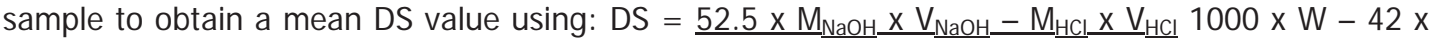
$\left(\mathrm{M}_{\mathrm{NaOH}} \times \mathrm{V}_{\mathrm{NaOH}}-\mathrm{M}_{\mathrm{HCl}} \times \mathrm{V}_{\mathrm{HCl}}\right)$ where $\mathrm{M}_{\mathrm{NaOH}}=$ molarity of $\mathrm{NaOH} \mathrm{V}_{\mathrm{NaOH}}=$ volume of $\mathrm{NaOH} \mathrm{M}_{\mathrm{HCl}}=$ molarity of $\mathrm{HCl}$ used to back titrate $\mathrm{NaOH} \mathrm{V}_{\mathrm{HCl}}=$ volume of $\mathrm{HCl}$ used to back titrate $\mathrm{NaOH} \mathrm{W}=$ sample weight $(\mathrm{g})$

\subsection{Preparation of the Adhesive Pastes}

Nine different dispersions of both native cellulose and $M C$ were prepared by adding distilled water $(5,10,15,20,25,30,35,40$ and $45 \% \mathrm{w} / \mathrm{v})$ and heating the dispersions to $45^{\circ} \mathrm{C}$ with continuous agitation for $15 \mathrm{~min}$. The slurries were then cooled to room temperature $\left(31^{\circ} \mathrm{C}\right)$ and allowed to form pastes.

\subsection{Determination of Tack Strength}

$10 \mathrm{~mL}$ of each paste was placed between two cardboard laminates measuring $15 \times 3 \mathrm{~cm}$ with bond area of $6 \times 2 \mathrm{~cm}$. One end of the laminates was clamped, the other end turned $180^{\circ}$ downwards and a pan attached with increasing standard masses added to the pan. The minimum load needed for the peel to begin was recorded.

\subsection{Rolling Ball Test}

Standard ASTM D 3121-94 method using $11.1 \mathrm{~mm}$ diameter steel ball was employed to determine the rolling ball distances of the pastes (Anon, 1997). 


\subsection{Determination of Gelation Time}

$20 \mathrm{~g}$ of each of the nine pastes was heated at $105^{\circ} \mathrm{C}$, cooled to room temperature $\left(31^{\circ} \mathrm{C}\right)$ and the time taken for it to form a firm gel was noted.

\subsection{Determination of Drying Time}

A uniform $150 \mu$ m-thick film of each paste was cast on two glass slides with their edges tapped off with a layer of masking tape. The drying time for each paste was determined by touching the film lightly with index finger at an interval of $2 \mathrm{~min}$. The first time the film was felt to be tacky enough, but do not transfer a wet film to the finger was recorded.

\subsection{Evaluation of Optical Clarity}

Light transmittance (\% T) of each of the nine pastes was determined at $650 \mathrm{~nm}$ against water blank using J asco V530 spectrophotometer (J asco Corporation, Tokyo, Japan) following standard method (Sharma, 2011).

\subsection{Determination of Relative Viscosity}

The efflux time (t) it takes $20 \mathrm{~cm}^{3}$ of each of the nine solutions to flow through an Ostwald viscometer was noted and related to the corresponding efflux time $\left(t_{0}\right)$ for $20 \mathrm{~cm}^{3}$ of water at room temperature $\left(33^{\circ} \mathrm{C}\right)$. The relative viscosity $\left(\dot{\eta}_{\text {rel }}\right)$ of each solution was then obtained from the expression: $\dot{\eta}_{\text {rel }}=\dot{\eta} / \dot{\eta}_{0}=\mathrm{t} / \mathrm{t}_{0}$ where $\dot{\eta}$ is the intrinsic viscosity of the test solution and $\dot{\eta}_{0}$ is the intrinsic viscosity of water, respectively. 10 minutes were allowed for the viscometer and test solution to attain thermal equilibrium.

\subsection{Assessment of Syneresis}

$5 \mathrm{~g}$ of each paste was transferred to a tube and refrigerated at $-20^{\circ} \mathrm{C}$ for $24 \mathrm{~h}$, and then thawed at room temperature for $6 \mathrm{~h}$. The tube was taken out time and centrifuged at 3000 revolutions per minute (rpm) for $20 \mathrm{~min}$ in L-550 centrifuge (Xiang-Yi Centrifuge Instrument Co. Ltd, Changsha, China). The water layer formed was decanted, the residual paste weighed and the percentage of water separated calculated using the formula:

$$
\text { Syneresis }=\frac{m_{2}-m_{3}}{m_{2}-m_{1}} \times 100 \%
$$

Where $m_{1}$ is the weight of centrifuge tube $(g), m_{2}$ is the weight of centrifuge tube and the starch paste $(\mathrm{g}), \mathrm{m}_{3}$ is the weight of centrifuge tube and the starch paste after centrifuging $(\mathrm{g})$.

\section{Results}

\subsection{Tack Strength and Rolling-Ball Distance}

The effect of degree of substitution of the eight etherified cellulose $(1.31,1.43,1.54,1.65,1.75$, $1.84,1.92$ and 98) and the control sample (0) on tack strength) and rolling-ball distance of the prepared adhesive pastes are shown in Figure 1. The minimum weight a bonded joint can support at a particular temperature before peeling (tack strength) of the nine samples ranged from $4.13 \mathrm{~g}$ (for the native cellulose) to $21.27 \mathrm{~g}$ (for the MC with highest DS value). This implies that for every 0.09 increase in DS, there is $2.14 \mathrm{~g}$ increase in tack strength. As tack strength is the most important 
parameter for choosing an adhesive for any use, the tack strength of the modified cellulose falls within the range recommended by standards bodies, such Adhesives and Sealants Council of the United States of America that recommends tack strength of a remoistenable adhesive to be within the range of 5 to $25 \mathrm{~g}$ as reported by Nolte et al (2009). The superior tack strength of the methyl cellulose in comparison to the native cellulose and the direct linear relationship between DS and tack strength can be attributed to increase in molecular weight. With respect to the rolling-ball distance (distance the $11.1 \mathrm{~mm}$ diameter steel ball moves before its movement is arrested) of the nine samples, it can be seen that their values decrease with increase in DS and ranged from $23.47 \mathrm{~cm}$ (native cellulose) to $5.79 \mathrm{~cm}$ (methyl chloride-containing cellulose with the highest DS value). These values roughly translate to a decrease of approximately $2.09 \mathrm{~cm}$ for every 0.09 increase in DS. The difference in the trends of tack strength and rolling-ball distance values is expected because an adhesive with greater tack strength is expected to have shorter rolling-ball distance (shorter distance the steel ball moves before its movement is arrested by the adhesive), is attributed to higher molecular weight of MC compared to that of the native cellulose. Methyl cellulose being $\mathbf{4 9 . 5}$ atomic mass units greater than the native cellulose, it is not surprising that methyl cellulose to have shorter rolling-ball distance value than the native cellulose.

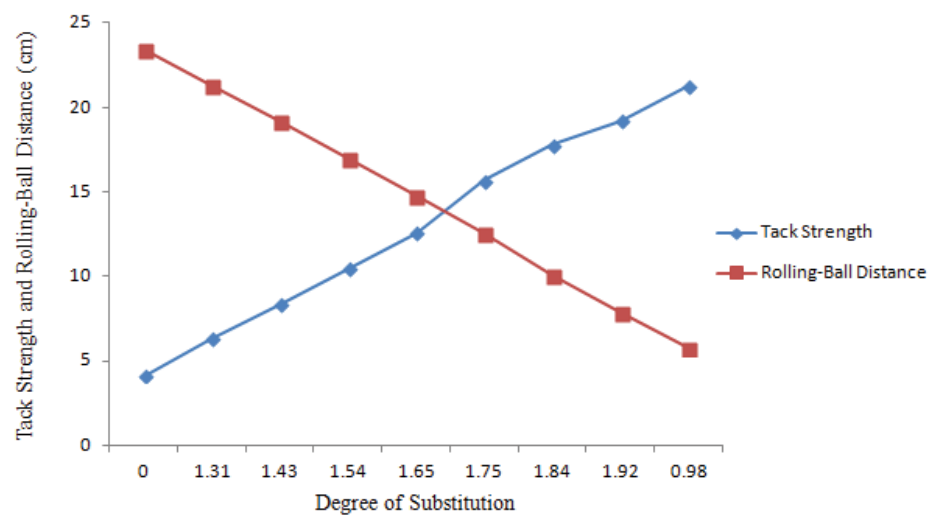

Fig. 3. Effect of Degree of Substitution on Tack Strength and Rolling-Ball Distance of the Native Cellulose and Methyl-Containing Cellulose Adhesive Pastes

\subsection{Drying and Gelation Times}

The effect of degree of substitution of the chemically modified cellulose (MC) and the native cellulose pastes on their drying and gelation times are shown in Figure 2. Drying time of the nine pastes ranged from $3.45 \mathrm{~min}$ for native cellulose to $45.83 \mathrm{~min}$ for the $M C$ and increase with increase DS value. Thus, for each 0.09 increase in DS, there is a corresponding $5.21 \mathrm{~min}$ increase in drying time. The gelation time of the samples ranged from $23.75 \mathrm{~min}$ (control) to $11.22 \mathrm{~min}$ (the most etherified). Thus, for each 0.09 increase in DS, there is a corresponding $1.57 \mathrm{~min}$ decrease in gelation time. As gel firmness is a marked physical feature of an ageing gel, gelation time is a convenient means of monitoring efficiency of an adhesive product. This is so because gel firmness is an undesirable sign of decreases in adhesive quality of cellulose-based products, especially adhesives. It can also be seen from Figure 2 that although the relation between DS and time of gel formation is linear, it is an inverse one. That is, as DS increase, gelation time decrease in similar proportion, in agreement with earlier findings (Maeda et al., 2002). If drying and gelation times of the chemically modified and native cellulose are compared, it can be observed that the drying time of $\mathrm{MC}$ is longer than that of native cellulose and increase with increase in DS value. Similarly, 
gelation time of the MC is less than that of native cellulose and decrease with increase in DS value. The inverse relation between gelation and drying times with increase in DS is expected because of increase in molecular size; molecular weight of $\mathrm{MC}$ is greater than that of native cellulose by about 50 atomic mass units. The inverse relation between gelation and drying times with increase in paste concentration is expected because thicker gels tend to take relatively longer time to dry, as found by Mario et al (2005) who studied the synthesis and characterization of sodium carboxymethyl cellulose from Cavendish Banana.

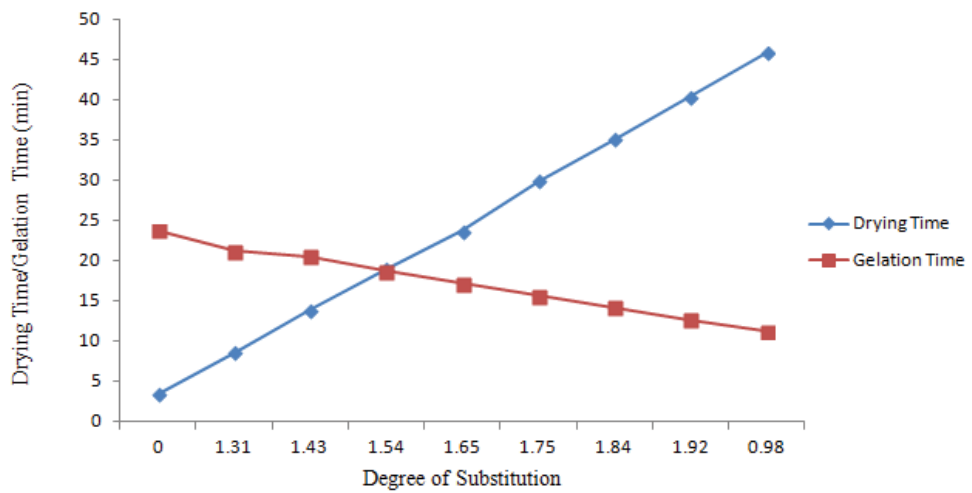

Fig. 4. Effect of Degree of Substitution on Drying and Gelation Times of the Native Cellulose and Methyl -Containing Cellulose Adhesive Pastes

\subsection{Optical Clarity, Relative Viscosity and Syneresis}

Optical clarity (expressed as percentage of light transmitted through a substance) that is, the quantity of light a paste allows to pass through it, relative viscosity (the relative ease with which a fluid sample flows in comparison with water as solvent) and syneresis (the amount of water seeped from or released by a substance) of the nine adhesive pastes (MC and native cellulose) are shown in Figure 3. From the figure, it can be seen that optical clarity decreases with increase in DS value of the methyl chloride-containing cellulose. The optical clarity of the samples ranged from 62.57 (native cellulose) to $22.34 \%$ (the MC with the highest value of DS), which translate to $5.03 \%$ decrease in transparency of the pastes for every 0.09 increase in DS value. The results implied that the pastes are not suitable for use as remoistenable adhesives where transparency is critical, in agreement with the findings of Amim et al (2009) who studied the solution behaviour of carboxymethylcellulose acetate butyrate. The relative viscosity of the samples, in contrast to optical clarity, increase with increase in DS value of the methyl chloride-containing cellulose and ranged from $18.73 \%$ (native cellulose) to $42.86 \%$ (the MC with the highest value of DS). This implies that there is $3.02 \%$ increase in relative viscosity of the methyl chloride-containing cellulose for every 0.09 increase in DS value. The inverse relationship between optical clarity and relative viscosity is expected because thicker adhesive (one with higher relative viscosity value) is expected to allow less light tp pass through it than a thinner one (having lower relative viscosity value). The syneresis values of the samples, like that of optical clarity can be seen to decrease with increase in DS value of the methyl chloride-containing cellulose. The syneresis values of the samples ranged from $18.57 \mathrm{~g}$ (native cellulose) to $2.06 \mathrm{~g}$ (the MC with the highest value of DS), meaning $2.06 \%$ decrease in the amount of water released for every 0.09 increase in DS value of the methyl chloridecontaining cellulose. The decrease in syneresis with in crease in DS is attributed to the higher water-holding capacity of modified cellulose in comparison to the native cellulose. Another contributing factor may be the increase in molecular weight as the native cellulose is etherified. 


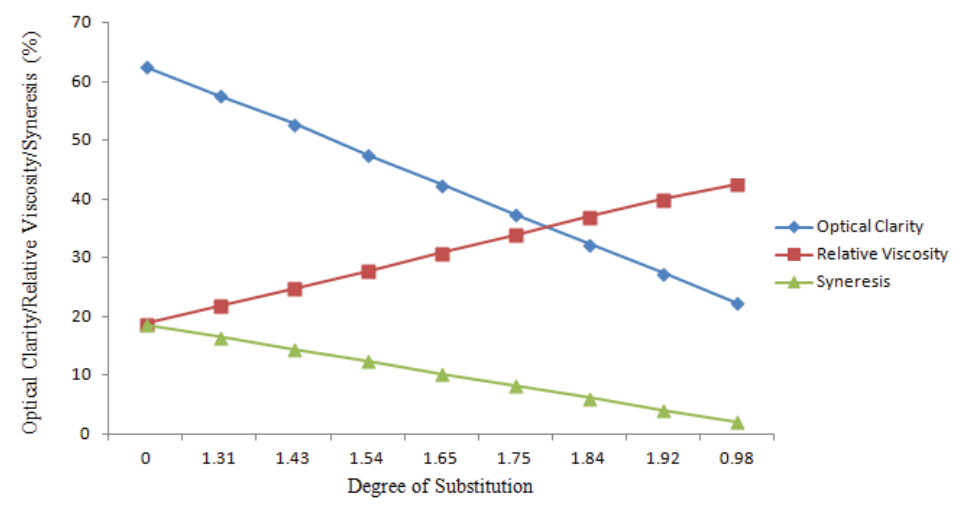

Fig. 5. Effect of Degree of Substitution on the Optical Clarity, Relative Viscosity and Syneresis of the Native Cellulose and Methyl-Containing Cellulose Adhesive Pastes

\section{Conclusion}

The results obtained from this work have shown that etherification of native cellulose has enhanced its adhesive properties and utilization in remoistenable applications, especially where transparency is not a critical parameter. Due to their relatively high bond strength, low syneresis biodegradability, renewability, environment friendliness and non-toxicity, production and use of MC from native cellulose in commercial scale is highly recommended. Nigeria stands a very good chance of improving her economy if cellulose can be sourced from non-edible plants and agricultural wastes and economically modified with safe and readily available reagents, such as methyl chloride.

\section{References}

Ahola, S., Salmi, J., Johansson, L. S., Laine, J. and Österberg, M. (2008). Model Films from Native Cellulose Nanofibrils. Preparation, Swelling, and Surface Interactions. Biomacromolecules 9: 12731282.

Amim, J., Petri, D. F. S., Maia, F. C. B. and Miranda, P. B. (2009). Solution behavior and surface properties of carboxymethylcellulose acetate butyrate. Cellulose 16: 773-782.

Anonymous (1997). American Society for Testing and Materials. ASTM Special Technical Publication No. 360, 123-134. Review of Methods for the Measurement of Tack, PAJ 1 Report No. 5.

Blackman, A., Bottle, S. Schmid, S., Mocerino, M. and Wille, U. (2012). Chemistry, $2^{\text {nd }}$ edn, John Wiley and Sons, Inc., New York, pp.977-1000.

Carey, F.A. (2008). Organic Chemistry, $7^{\text {th }}$ edn, McGraw-Hill Higher Education, New York, pp.1022-1061.

Carraher, J r, C.E. (2010). Introduction to Polymer Chemistry, $2^{\text {nd }}$ edn, CRC Press Boca Raton, pp. 85-140.

Chen, N., Maeda, N., Tirrell, M. and Israelachvili, J. (2005). Adhesion and Friction of Polymer Surfaces: The Effect of Chain Ends. Macromolecules 38: 3491-3503.

Doraiswamy, A., Dunaway, T. M., Wilker, J. J. and Narayan, R. J. (2009). Inkjet Printing of Bioadhesives. Journal of Biomedical Materials Research 89: 28-35.

Ebnesajjad, S. (2010). History of Adhesives. Handbook of Adhesives and Surface Preparation: Technology, Applications and Manufacturing. Elsevier, Amsterdam, p.137-154.

Elomaa, M. (2004). Determination of the degree of substitution of acetylated starch by hydrolysis, $1 \mathrm{H}$ NMR and TGA/IR. Carbohydrate Polymers 57: 261-267.

Eriksson, M., Notley, S. M. and Wågberg, L. (2007). Cellulose Thin Films: Degree of Cellulose Ordering 
and Its Influence on Adhesion. Biomacromolecules 8: 912-919

Forsström, J., Eriksson, M. and Wågberg, L. (2005). A new technique for evaluating ink cellulose interactions: initial studies of the influence of surface energy and surface roughness. Journal of Adhesion Science and Technology 19: 783-798.

Fox, S.C., Li, B., Xu, D. and Edgar, K.J. (2011). Regioselective esterification and etherification of cellulose: a review 12(6): 1956-1972.

Henriksson, M., Berglund, L. A., Isaksson, P., Lindström, T. and Nishino, T. (2008). Cellulose nanopaper structures of high toughness. Biomacromolecules 9: 1579-1585.

Hess, A.M. and Sullivan, D.L. (2005). Potential for toxicity with use of bitter orange extract and guarana for weight loss. The Annals of pharmacotherapy 39(3): 574-585.

Huang, Y. Y., Zhou, W., Hsia, K. J., Menard, E., Park, J-U., Rogers, J. A. and Alleyne, A. G. (2005). Stamp Collapse in Soft Lithography. Langmuir 21(17): 8058-8068.

Ito, T., Yeo, Y., Highley, C.B., Bellas, E.. Benitez, C.A. and Kohane, D.S. (2007). The prevention of peritoneal adhesions by in situ cross-linking hydrogels of hyaluronic acid and cellulose derivatives. Biomaterials 28: 975-983.

Jordan, S., Murty, M. and Pilon, K. (2004). Products containing bitter orange or synephrine: suspected cardiovascular adverse reactions. Canadian Medical Association Journal 171(8): 356-367.

Karabulut, E. and Wågberg, L. (2011). Design and characterization of cellulose nanofibrilbased freestanding films prepared by layer-by-layer deposition technique. Soft Matter 7: 3467-3474.

Klemm, D., Heublein, B., Fink, H. P. and Bohn, A. (2005) Cellulose: Fascinating biopolymer and sustainable raw material. Angew. Chemistry-International Edition 44: 3358-3393.

Maeda, N., Chen, N., Tirrell, M. and Israelachvili, J.N. (2002). Adhesion and Friction Mechanisms of Polymer-on-Polymer Surfaces. Science 297 (5580): 379-382.

Mario, P., Adinugraha, D.W. and Haryadi, M. (2005). Synthesis and characterization of sodium carboxymethyl cellulose from Cavendish Banana Psedostem (Musa cavendishii Lambert), Carbohydrate Polymers 62: 164-169.

McMurry, J. E. (2012). Organic Chemistry, Brooks/Cole Cengage Learning, Belmont, pp.1001-1034.

Mittal, K.L. and Pizzi, A. (2003). Historical Development of Adhesives and Adhesive Bonding. Handbook of Adhesive Technology (2nd ed., revised and expanded), Marcel Dekker, Inc., New York, p.11-32.

Nolte, A. J., Chung, J. Y., Walker, M. L. and Stafford, C. M. (2009). In situ Adhesion Measurements Utilizing Layer-by-Layer Functionalized Surfaces. ACS Appl. Mater. Interfaces 1: 373-380.

Notley, S. M. and Norgren, M. (2006). Measurement of interaction forces between lignin and cellulose as a function of aqueous electrolyte solution conditions. Langmuir 22: 11199-11204.

Pettersson, T. and Dedinaite, A. (2008). Normal and friction forces between mucin and mucin-chitosan layers in absence and presence of SDS. Journal of Colloid Interface Sciience 324: 246-256.

Pushpamalar, V., Langford, S. J., Ahmad, M. and Lim, Y. Y. (2006). Optimization of reaction conditions for preparing carboxymethyl cellulose from sago waste. Carbohydrate Polymers 64: 312 -318.

Rodrigues, M., Alves, G. and Falcão, A. (2013). Investigating herb-drug interactions: the effect of Citrus aurantium fruit extract on the pharmacokinetics of amiodarone in rats. Food Chemistry and Toxicology 60: 153-159.

Samios, E., Dart, R. K. and Dawkins, J. V. (1997) Preparation, characterization and biodegradation studies on cellulose acetates with varying degrees of substitution. Polymer 38: 3045-3054.

Sharma, B.K. (2011). Industrial Chemistry, Krishna Prakashan Media (P) Ltd, Meerut, pp. pp.1371-1380

Villetti, M.A., Bica, C.I., Garcia, I.T., Pereira, F.V., Ziembowicz, F.I., Kloster, C.L. and Giacomelli, C. (2011). Physicochemical properties of methylcellulose and dodecyltrimethylammonium bromide in aqueous medium. Journal of Physical Chemistry Part B 115(19): 5868-5876. 